\title{
Combined Immunotherapy and Targeted Treatment for Primary Alveolar Soft part Sarcoma of the Lung $\rrbracket$ Case Report and Literature Review
}

Hui Su ( $\nabla$ shhappy065010733@126.com )

Liaocheng People's Hospital https://orcid.org/0000-0002-5174-8217

Chao Yu

Liaocheng People's Hospital

Xuezhen Ma

Qingdao Center Hospital: Qingdao Center Medical Group

Qing Cui Song

Liaocheng People's Hospital

\section{Research Article}

Keywords: Primary acinar soft part sarcoma of the lung, Anlotinib, Camrelizumab, Targeted therapy, Immunotherapy

Posted Date: February 12th, 2021

DOl: https://doi.org/10.21203/rs.3.rs-190035/v1

License: (c) (i) This work is licensed under a Creative Commons Attribution 4.0 International License. Read Full License

Version of Record: A version of this preprint was published at Investigational New Drugs on March 25th, 2021. See the published version at https://doi.org/10.1007/s10637-021-01105-6. 
Combined immunotherapy and targeted treatment for primary alveolar soft part sarcoma of the lung: Case report and literature review

Hui Su${ }^{1}$, Chao Yu ${ }^{2}$, Xuezhen $\mathrm{Ma}^{3}$, Qingcui Song ${ }^{1}$

${ }^{1}$ Author affiliations: Department of Oncology, Liaocheng People's Hospital, Liaocheng 252000, Shandong Province, China. tel:+8619963506113, Email: shhappy065010733@126.com

${ }^{2}$ Author affiliations: Department of Orthopedics, Liaocheng People's Hospital, Liaocheng 252000, Shandong Province, China

${ }^{3}$ Author affiliations: Department of Oncology, Qingdao Central Hospital, The Second Affiliated Hospital of Medical College of Qingdao University, Qingdao 266000, Shandong, China

Correspondence author: Hui Su, Department of Oncology, Liaocheng People's Hospital, Liaocheng 252000, Shandong Province, China. tel:+8619963506113,Email: shhappy065010733@126.com

\begin{abstract}
Primary acinar soft part sarcoma of the lung is a rare malignancy with unique cellular structure and clinical and genetic characteristics. Most patients have no obvious clinical symptoms, and only a few develop respiratory symptoms. The typical histological characteristics are acinoid or organ-like structure. Immunofluorescence in situ hybridization suggests a rearrangement of the transcription factor E3 gene. Patients with primary acinar soft part sarcoma of the lung respond poorly to chemotherapy and are thus primarily treated with surgery and targeted therapy. We report herein a unique case of primary alveolar soft part sarcoma of the lung. The patient was a 24-year-old man who underwent targeted treatment combined with immunotherapy. Some of the lesions completely disappeared after treatment, indicating significant efficacy. The treatment benefit observed in the current case provides preliminary evidence that targeted therapy combined with immunotherapy maybe a safe and effective modality in patients with primary pulmonary ASPS, thus warranting further investigation.
\end{abstract}

Key words: Primary acinar soft part sarcoma of the lung, Anlotinib, Camrelizumab, Targeted therapy, Immunotherapy

\title{
Case summary
}

The patient was a 24-year-old male who visited Shandong Provincial Thoracic Hospital for hemoptysis for 2 days in February 2017. Purified protein derivative, tuberculosis antibody, T-SPOT-TB, and other examinations showed tuberculosis, and the patient was accordingly prescribed with anti-tuberculosis and hemostatic treatment. The patient also underwent bronchoscopy, but the family did not follow up the result. In June 2018, he developed headache, nausea, vomiting, anorexia, and fatigue. Cranial magnetic resonance imaging (MRI) showed multiple shots. A retrospective review of the bronchoscopy image showed tumors on the right lower lung, and positron emission tomography/computed tomography (PET/CT) showed malignant lung tumors and widespread metastasis to the lungs, brain, pleura, and right femur. Abdominal B-ultrasound also showed multiple pancreatic metastasis. The pathological diagnosis was pulmonary alveolar soft tissue sarcoma. On immunohistochemistry performed on September 9, 2010, the tumor stained positively for TFE (3+) and negatively for CK8/18, EMA, HMB45, Melam-A, S-100, S/n, CgA, MYOD, and Myogenin. Written informed consent to publish the case details and any accompanying images was obtained from the patient.

\subsection{Therapetic process}

Whole-brain radiotherapy (DT: $39 \mathrm{~Gy} / 13 \mathrm{~F}$ ) was started on September 14, 2019. This was followed by 2 cycles of systemic chemotherapy (ifosfamide ( $2 \mathrm{~g}, \mathrm{~d} 1-5)$ + doxorubicin (60 mg, d1-3)) combined with apatinib (0.5 g, qd). However, chemotherapy was suspended because of grade IV thrombocytopenia, while apatinib was continued for 11 months. Treatment response was evaluated as stable disease (SD) based on CT findings. However, chest and abdominal CT on August 23, 2019 showed multiple liver metastases, and the patient was determined to have progressive disease (PD). Apatinib was switched to anlotinib (12 mg, d1-14, q3w) for 2 months. Chest and abdominal CT on October 23, 2019 showed progression in lung lesion (indicating progressive disease (PD)). As such, immunotherapy (camrelizumab $200 \mathrm{mg}$ d1 q2w + anlotinib 12 mg d1-14 q3w) was added as treatment until on December, 2020. Multiple evaluations were performed during combination therapy showed that the pancreatic and liver lesions disappeared (indicating complete 
response (CR)) and that the lung and brain lesions were significantly reduced (indicating partial response (PR)). Blood and immune indicators (including blood routine; biochemical, coagulation, myocardial, and thyroid function markers; brain natriuretic peptide; and troponin I) were evaluated before every treatment cycle. Blood indicators were also evaluated every two cycles. In addition, electrocardiogram and cardiac color doppler ultrasound were performed. Reactive cutaneous capillary endothelial proliferation (RCCEP) occurred after 1 cycle of camrelizumab. The patient was prescribed with some drugs by a consulting dermatologist. No further significant increase in the rash was observed during treatment. And, immune hepatitis toxic reaction occurred after 6 cycles of camrelizumab, and thus the drug was stopped for 1 month. Liver function returned to normal after liver protection and enzyme reduction drugs were administered. However, chest and abdominal CT and brain MRI revealed new liver, pancreas, and brain lesions(PD) on December 12, 2020.

\subsection{Efficacy evaluation}

Lung lesions: Before treatment, multiple metastases were noted in both lungs, with a maximum tumor diameter of $3.5 \mathrm{~cm}$. After chemotherapy + apatinib treatment, the maximum tumor diameter was reduced by $28 \%$ to $2.5 \mathrm{~cm}$, indicating SD. Before monoclonal antibody treatment with anlotinib + camrelizumab, the maximum tumor diameter was $2.7 \mathrm{~cm}$; this was reduced by $70 \%$ to $0.8 \mathrm{~cm}$ posttreatment.

Brain lesions: Before treatment, multiple metastatic lesions were noted in the left cerebellar hemisphere, bilateral occipital lobe, left frontal lobe, right lateral ventricle next to the body, and left parietal lobe, with the largest located in the left frontal lobe and cerebellar hemisphere (maximum tumor diameter: $2.7^{*} 2.6^{*} 2.8 \mathrm{~cm}$ ). After treatment with whole brain radiotherapy + chemotherapy + apatinib, the maximum tumor diameter was reduced by $42 \%$ to $1.4 * 1.5 * 1.6 \mathrm{~cm}$, indicating PR. Before anlotinib + camrelizumab treatment, the maximum tumor diameter was 1.5*1.0*1.2. Punctate enhancement, indicating PR, was observed after treatment.

Pancreatic lesions: Before treatment, multiple metastases, with a maximum tumor diameter of $2.6 \mathrm{~cm}$, were noted. Treatment with chemotherapy + apatinib reduced the maximum diameter to $2.4 \mathrm{~cm}$, indicating SD. Before anlotinib + camrelizumab treatment, the maximum diameter was $3.8 \mathrm{~cm}$. The pancreatic lesions disappeared posttreatment, indicating $\mathrm{CR}$.

Liver lesions: Before treatment with anlotinib + camrelizumab, multiple metastases, with a maximum tumor diameter of $1.0 \mathrm{~cm}$, were noted. The liver lesions disappeared posttreatment, indicating CR.
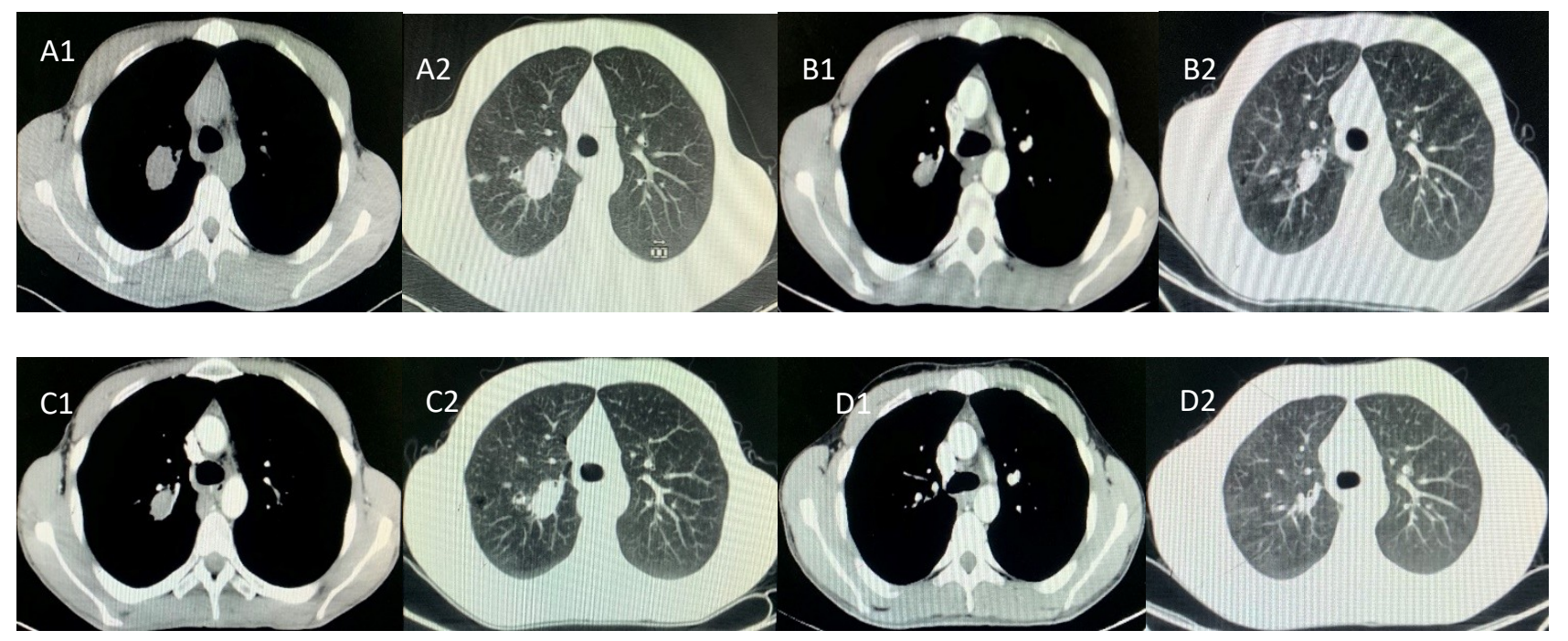

Figure 1. Dynamic changes in chest CT imaging during treatment

A1、 2 Before treatment (2018-08-31)

B1、 2 After chemotherapy + apatinib treatment (2019-05-28)

C1 、 2 Before anlotinib + camrelizumab treatment ( 2019-10-24) D1 、 2 After anlotinib + camrelizumab treatment (2020-9-27) 

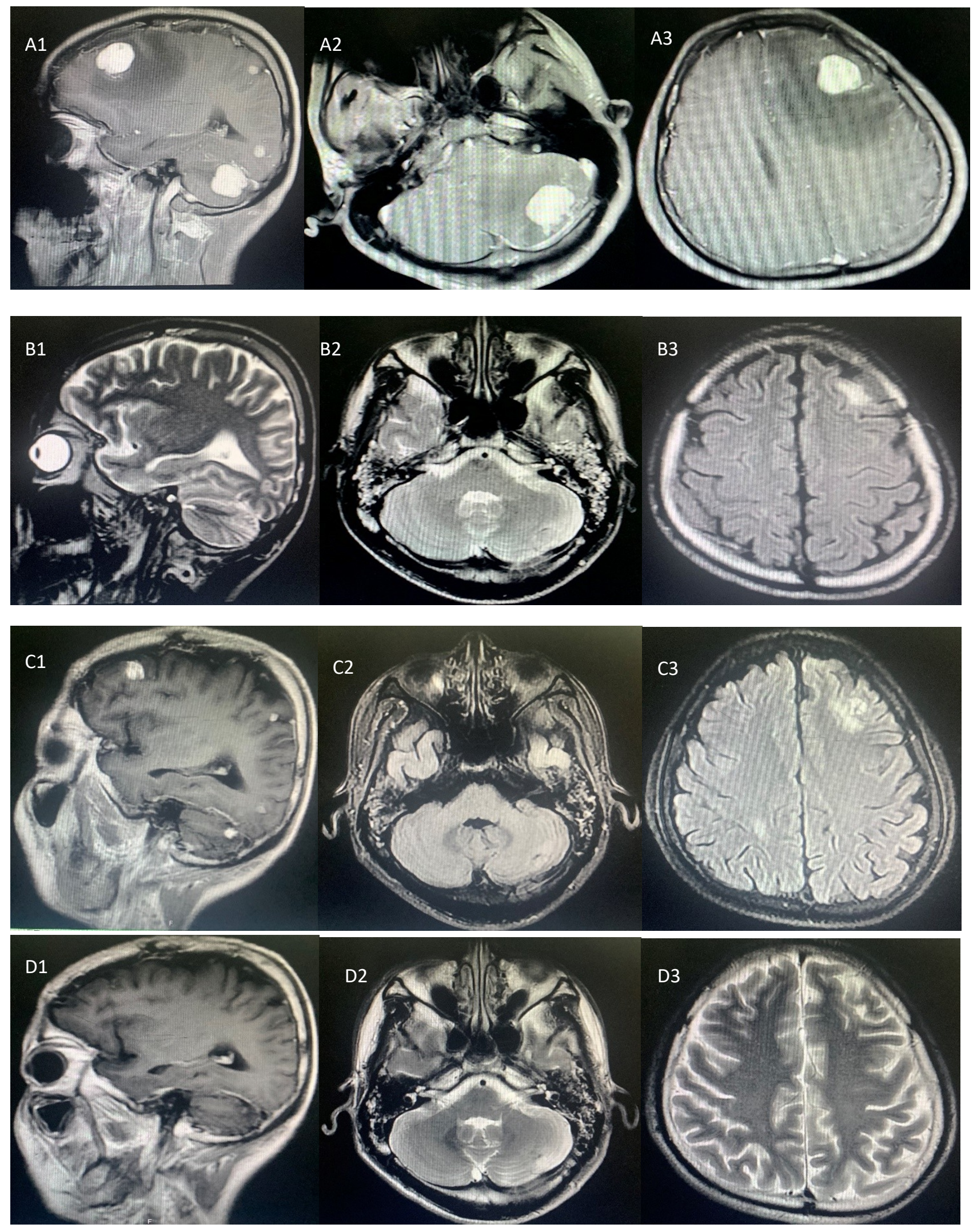

Figure 2. Dynamic changes in brain MRI imaging during treatment

A1、2、3 Before treatment (2018-08-31)

B1、2、3 After chemotherapy + apatinib treatment (2019-05-

28)

C1 、 2、 3 Before anlotinib + camrelizumab treatment (2019-10-24) D1 $2 、 3$ After anlotinib + camrelizumab treatment (2020-9-27) 


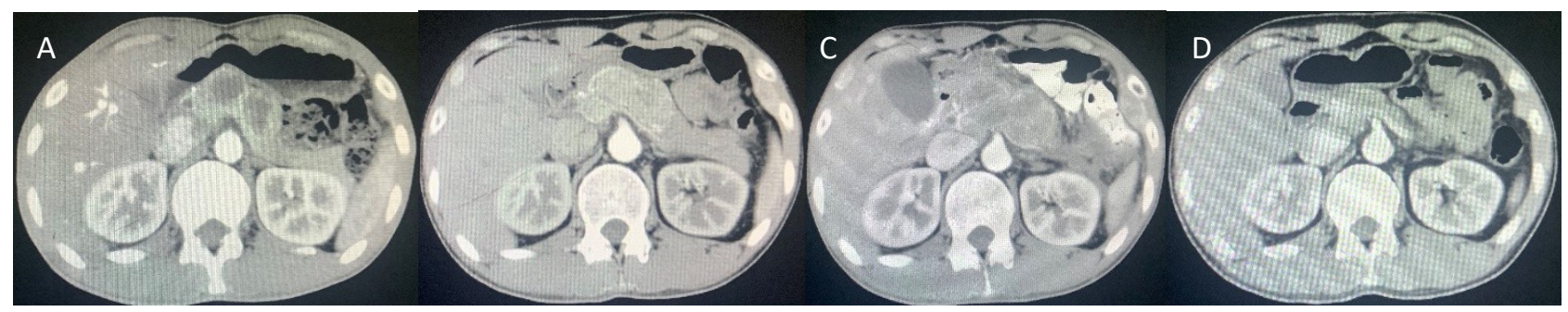

Figure 3. Dynamic changes in CT imaging of the pancreas during treatment
A Before treatment (2018-10-4)
B After chemotherapy + apatinib treatment (2019-05-28)

C Before anlotinib + camrelizumab treatment (2019-10-24) D After anlotinib + camrelizumab treatment (2020-9-27)

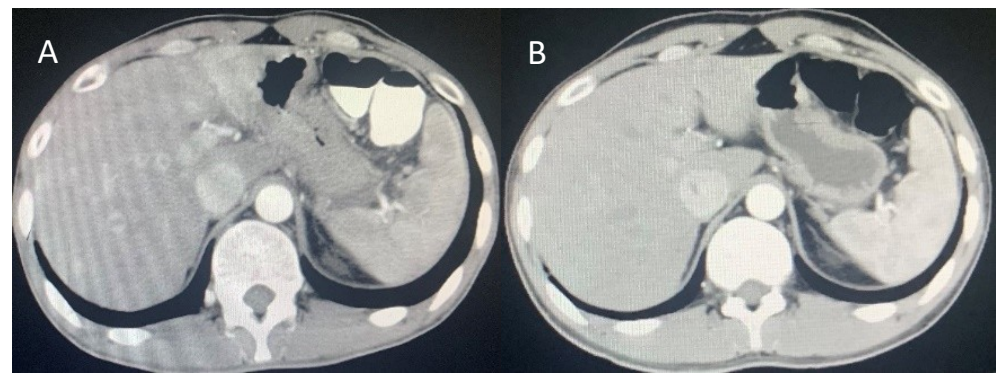

Figure 4. Dynamic changes in CT imaging of the liver during treatment

A Before anlotinib + camrelizumab treatment (2019-10-24) B After anlotinib + camrelizumab treatment (2020-927)

\section{Discussion}

Acinar soft part sarcoma (ASPS) is a soft tissue malignant tumor of unknown origin, with the cells and tissues presenting with alveolar or organ-like arrangement. ASPS was first reported by Christopherson et al ${ }^{[1]}$ in 1952 . It occurs in children and adolescents, accounting for about $1 \%$ of all soft tissue sarcomas ${ }^{[2]}$. ASPS develops gradually, and it has a predilection for the head and neck, particularly the orbits and tongue. Among children. In adults, the tumors mostly develop in the deep soft tissues of the limbs, especially in the thighs and buttocks. Some tumors can also arise in rare areas such as the female reproductive tract, breast, and meninges. Given its insidious onset, some patients present with extensive metastases, such as to the lungs, brain, and bones, on diagnosis. The origin of ASPS remains unclear to date; however, many scholars support the concept of myogenicity. The diagnosis of ASPS mainly relies on typical pathological features and immunophenotypes. Molecular genetic studies of ASPS have confirmed ectopic $t(X ; 17)(P 11 ; 25)$, causing the ASPSA key region gene 1 (ASPSACR-1) and transcription enhancer TFE3 on the 17q25 chromosome to produce a functional fusion gene, that is, ASPSACR1-TFE3 ${ }^{[3]}$. As such, immunofluorescence in situ hybridization and real-time polymerase chain reason to detect E3 gene rearrangement and E3 gene fusion, respectively, are useful diagnostic modalities ${ }^{[4]}$. Pulmonary primary ASPS needs to be differentiated from acinar rhabdomyosarcoma, metastatic hepatocellular carcinoma, paraganglioma, large cell neuroendocrine tumors, and other diseases. The pathological and immunohistochemical characteristics of the current case are consistent with those reported in the literature. The patient was a young male with no previous history and other soft tissue primary lesions. The diagnosis of primary pulmonary ASPS was confirmed by combining imaging findings and typical histopathological features.

Clinically, pulmonary metastatic ASPS is more common than pulmonary primary ASPS. Only four cases of primary pulmonary ASPS have been reported in PubMed to date ${ }^{[5-8]}$. To our best knowledge, the current case is only the fifth case to be reported. With respect to prognosis, the 5-year survival rate of ASPS patients is about 56\%. Advanced age, distant metastasis at the time of diagnosis, and tumor diameters measuring $>10 \mathrm{~cm}$ adversely influence prognosis ${ }^{[9]}$. ASPS primarily involves large tumor resection. The 5-year survival rate of patients with localized ASPS who undergo surgical resection is approximately $80 \%$, whereas that of patients who do not undergo resection is only $10 \%{ }^{[9]}$. ASPS is not sensitive to traditional radiotherapy and chemotherapy ${ }^{[10-13]}$; chemotherapy only has an efficacy of less than $10 \%{ }^{[13]}$. Meanwhile, the benefit of radiotherapy on ASPS is controversial. Some studies reported that radiotherapy can control local recurrence and delay distant metastasis while some studies also reported that radiotherapy has not beneficial for ASPS ${ }^{[14-16]}$. Our patient had multiple brain and lung metastases at the time of diagnosis, and thus there was no opportunity for surgery. However, the brain and lung lesions were reduced by $42 \%$ and $28 \%$, respectively, after total brain radiotherapy and 2 cycles 
of chemotherapy combined. This indicates the benefit of radiotherapy and chemotherapy on primary lung ASPS.

Anlotinib is an oral multi-target tyrosine kinase inhibitor (TKI) that exerts its anti-tumor effects by acting on various kinases. It also has high selectivity for vascular endothelial growth factor (VEGFR)-2 and VEGFR-3. On June 2019, anlotinib became the first soft tissue sarcoma (STS)-targeted drug to be approved in China as first-line treatment for ASPS and as second-line treatment for other advanced STS. A multi-center, single-arm phase II clinical trial (NCT01878448) ${ }^{[17]}$ studied the efficacy of anlotinib in 166 patients with advanced STS who failed conventional treatment. Anlotinib showed good clinical efficacy. The 12-week progression-free survival (PFS) rate was $68 \%$, the mPFS was 5.63 months, and the overall response rate (ORR) was $11.45 \%$. The ASPS group ( $n=13,12 \mathrm{mg} \mathrm{qd}$ ) had the highest ORR at $46 \%$ ( 6 cases achieved PR); the 12-week PFS rate was 77\%; mPFS, 21 months; and the median overall survival (mOS) was not reached. In 2018, ASCO reported the results of their phase IIb clinical study of anlotinib for second-line treatment of STS (ALTER 0203) ${ }^{[18]}$. The $\mathrm{mPFS}$ of the anlotinib group was significantly higher than that of the placebo group (18.23 vs 3 months, $\mathrm{P}<0.0001$ ).

Other targeted drugs can also be used for ASPS treatment. For example, the National Comprehensive Cancer Network (NCCN) guideline recommends sunitinib as the first-line treatment for ASPS ${ }^{[19]}$. Paulina et al ${ }^{[20]}$ reported that among their 15 ASPS patients with lung metastases who were treated with sunitinib, 6, 8, and 1 patient achieved PR, SD, and PD, respectively. The clinical benefit rate was $93 \%$, and the mPFS was 19 months. Li et al ${ }^{[21]}$ retrospectively evaluated 14 ASPS patients who were ineligible for resection or had metastases. The treatment response to sunitinib was PR in 4 patients and SD in 10 patients. The mPFS was 41 months, and the mOS was not reached. In a follow-up study of 15 patients with advanced ASPS ${ }^{[22]}$, the mOS and the 5 -year OS after sunitinib treatment was 56 months and could reach $49 \%$. The mPFS was 19 months, and the 5-year PFS rate was 30\%. In total, 6 and 8 of the patients achieved PR and SD, respectively, and 1 patient developed PD. Another targeted drug is pazopanib, which was approved by the US FDA and the European Union in 2012 for the treatment of advanced STS in adults ${ }^{[23]}$. However, because pazopanib cannot penetrate the blood-brain barrier, its application in patients with brain metastases ASPS is limited ${ }^{[24]}$. In a study of 6 patients with metastatic ASPS ${ }^{[25]}$, 1 and 5 patients achieved PR and SD, respectively. Within a median follow-up time of 33 months, the mPFS was 5.5 months, the mOS was not reached, and the trial reached the primary endpoint. Another retrospective analysis ${ }^{[26]}$ of 29 evaluable patients reported CR, PR, SD, and PD in 1, 7, 17, and 4 patients, respectively. The median follow-up was 19 months, the mPFS was 13.6 months, the 1-year PFS was 59\%, and the mOS was not reached. Based on these findings, pazopanib was recommended as the primary drug for systemic treatment of ASPS (Class 2A evidence) in the 2019 NCCN guidelines. Cediranib is a multi-target small molecule TKI. In a double-blind, randomized, phase II clinical trial by Judson et al ${ }^{[27]}$, the median percentage change in the sum of the target marker lesion for the evaluable population was significantly higher in the cediranib arm than that in the placebo arm (-8.3\% (IQR: -26.5 to 5.9 ) vs $13.4 \%$ (IQR: 1.1 to 21.3), one-sided $\mathrm{p}=0.0010$ ). Meanwhile, a follow-up exploratory phase II clinical trial of cediranib for the treatment of children with metastatic ASPS did not meet the expected ORR of the trial ${ }^{[28]}$. Two retrospective analyses ${ }^{[29-30]}$ reported satisfactory results in patients with advanced ASPS treated with apatinib. In addition, in a clinical trial of 40 MET-positive ASPS patients, the disease control rate of crizotinib treatment reached $87.5 \%{ }^{[30]}$.

Immune checkpoint inhibitors, such as the CTLA-4 monoclonal antibodies and PD-1 monoclonal antibodies, were first suggested to be effective treatments for drug-resistant ASPS by Conley in [indicate the year here ${ }^{[31]}$. Camrelizumab is a humanized immunoglobulin G4 monoclonal antibody ${ }^{[32]}$ that can target PD-1 to block the interaction between PD-L1 and programmed death ligand 2, ultimately playing an anti-tumor effect ${ }^{[33]}$. The NCT02961101, NCT03250962, and NCT03155425 trials ${ }^{[34-35]}$ all showed the efficacy of camrelizumab for classic Hodgkin's lymphoma (CHL). Accordingly, camrelizumab was officially approved by the National Medical Products Administration (NMPA) on May 2019 for the treatment of relapsed or refractory CHL after at least second-line system therapy ${ }^{[36]}$. Although there is currently no clinical trial on camrelizumab for ASPS, the drug is used in many other solid tumors, such as osteosarcoma ${ }^{[37]}$, esophageal cancer ${ }^{[38-39]}$, gastric cancer ${ }^{[40]}$, liver cancer ${ }^{[41-42]}$, lung cancer ${ }^{[43]}$, and nasopharyngeal cancer ${ }^{[44]}$. Its safety and effectiveness will be further validated in series of large-scale clinical trials. The common adverse reactions of camrelizumab include RCCEP ${ }^{[34-35]}$, elevated blood bilirubin, altered liver function ${ }^{[40]}$, fever, fatigue, hypothyroidism, proteinuria, and lung infections. Most of the adverse reactions of camrelizumab are reversible, with prompt diagnosis being instrumental ${ }^{[45]}$. Patients commonly chose camrelizumab over the more expensive pambrolizumab or nivolumab. In the current case, RCCEP and liver function abnormalities developed during the therapy, but these were successfully controlled through active drug treatment. Accordingly, camrelizumab was continued, and good results were achieved. 
Other immunological drugs for ASPS have also been reported. Wilky et al ${ }^{[46]}$ conducted a randomized, single-arm, phase II clinical trial of axitinib combined with pembrolizumab for sarcoma patients. The 3-month PFS rate was 72.7, and the mPFS of the patients with ASPS was higher than that of the patients with other sarcomas (12.4 months vs 3.0 months). In the current case, anlotinib combined with camrelizumab showed high clinical effectiveness. The pancreatic and liver lesions disappeared, and the brain and lung lesions were significantly reduced. The PFS was 14 months, which was similar to those reported in the literature. Notably, pembrolizumab was added in the 2019 edition of the NCCN guidelines (2B recommendation). Lewin et al ${ }^{[47]}$ compared durvalumab monotherapy with combination treatment with durvalumab and tremelimumab to treat 2 patients with advanced ASPS. PD occurred after 12 months in the single-agent arm, while PR was achieved in the combination treatment arm, with the tumor volume reduced by $73 \%$. Further, the duration has exceeded 18 months. In addition, they conducted genome analysis of ASPS and found that immune checkpoint inhibitors may be an effective treatment strategy for ASPS patients. Toripalimab is a recombinant humanized anti-PD-1 monoclonal antibody independently developed in China. In a phase I clinical trial (JS001) of toripalimab for advanced or refractory ASPS, 1 and 2 of the 12 patients achieved CR and PR, respectively. The mPFS is expected to be 12.4 months ${ }^{[48]}$.

\section{Conclusion}

Primary pulmonary ASPS can recur and metastasize, and thus imaging and follow-up are necessary. Although surgery remains the optimal treatment strategy, targeted therapy combined with immunotherapy may be a more effective approach for primary ASPS patients with advanced distant metastasis. The treatment benefit observed in the current case provides preliminary evidence that targeted therapy combined with immunotherapy maybe a safe and effective modality in patients with primary pulmonary ASPS, thus warranting further investigation.

Ethical approval: The case report comply with the current laws of the country in which he was performed.

Informed consent: We obtained informed consent from the patient in this case report.

Consent for publish: Not applicable.

Availability of data and material : Not applicable.

Competing interests: Hui Su declares no conflict of interest, Chao Yu declares no conflict of interest, Xuezhen Ma declares no conflict of interest, Qingcui Song declares no conflict of interest.

Funding : No.

Authors' contributions: Hui Su and Qingcui Song drafted the manuscript and the collation of the case. Xuezhen Ma critically revised the paper. Chao Yu participated in the translation of the article. All authors contributed toward data analysis, drafting and revising the paper and agree to be accountable for all aspects of the work.

Acknowledgements: The authors thank the patient for his participation in this study and for the patients' agreement to the publication of the report.

\section{References}

[1] Christopherson WM, Foote FW, Stewart FW. Alveolar soft part sarcomas; structurally characteristic tumors of uncertain histogenesis [J]. Cancer, 1952, 5(1): 100111.

[2] SOHEILIFAR M H, TAHERI R A, ZOLFAGHARI EMAMEH R, et al. Molecular landscape in alveolar soft part sarcoma:implications for molecular targeted therapy[J]. Biomed Pharmacother, 2018, 103:889-896.

[3]Mitton B, Federman N. Alveolar soft part sarcomas: molecular pathogenesis and implications for novel targeted therapies [J]. Sarcoma,2012:428789.

[4] Paoluzzi L, Maki RG. Diagnosis, Prognosis, and Treatment of Alveolar Soft Part Sarcoma: a Review[J]. JAMA Oncol, 2019, 5(2):254 260 .

[5] Trabelsi A, Ben Abdelkrim S, Taher Yacoubi M, et al. Sarcome alvéolaire primitif du poumon[J]. Rev Mal Respir, 2009, 26(3):329 332.

[6] Zhao M, Rao Q, Wu C, et al. Alveolar soft part sarcoma of lung: report of a unique case with emphasis on diagnostic utility of molecular genetic analysis for TFE3 gene rearrangement and immunohistochemistry for TFE3 antigen 
expression[J]. Diagn Pathol, 2015, 10: 160.

[7] Tsutsumi Y, Deng YL. Alveolar soft part sarcoma of the pulmonary vein[J]. Acta Pathol Jpn, 1991, $41(10): 771777$.

[8] Kim YD, Lee CH, Lee MK, et al. Primary alveolar soft part sarcoma of the lung[J]. J Korean Med Sci, 2007,22 (2): 369 372.

[9] Wang H, Jacobson A, Harmon DC, et al. Prognostic factors in alveolar soft part sarcoma: a SEER analysis[J]. J Surg Oncol, 2016, 113(5):581 586.

[10] JAGODZIŃSKA-MUCHA P,ŚWITAJ T, KOZAK K, et al.Long-term results of therapy with sunitinib in metastatic alveolar soft part sarcoma[J]. Tumori, 2017, 103(3):231-235.

[11] ORBACH D, BRENNAN B, CASANOVA M, et al. Paediatric and adolescent alveolar soft part sarcoma:a joint series from European cooperative groups[J]. Pediatr Blood Cancer, 2013,60(11):1826-1832.

[12] MENG N, ZHANG X, LIAO A, et al. Management of recurrent alveolar soft-part sarcoma of the tongue after external beam radiotherapy with iodine-125 seed brachytherapy[J]. Head Neck, 2014, 36(12):E125-E128.

[13] SPARBER-SAUER M, SEITZ G, VON KALLE T, et al .Alveolar soft-part sarcoma: primary metastatic disease and metastatic relapse occurring during long-term follow-up[J].Pediatr Blood Cancer, 2018, 65(12):e27405.

[14] Falkensternge R F, Kimmich M, Wohlleber M,et al. Lung metastasis of primary alveolar soft-part sarcoma occurring 20 years after initial treatment[J].Case Rep Oncol Med,2013,2013(1):690520.

[15] Brennan B, Zanetti I, Orbach D, et al. Alveolar soft part sarcoma in children and adolescents: the European Paediatric Soft Tissue Sarcoma study group prospective trial (EpSSG NRSTS 2005) [J]. Pediatr Blood Cancer, 2018, 65(4).

[16] Flores RJ, Harrison DJ, Federman NC, et al. Alveolar soft part sarcoma in children and young adults: a report of 69 cases[J]. Pediatr Blood Cancer, 2018, 65(5): e26953.

[17] CHI Y, FANG Z, HONG X, et al. Safety and efficacy of anlotinib, a multikinase angiogenesis inhibitor, in patients with refractory metastatic soft tissue sarcoma [J]. Clin Cancer Res, 2018, 24 （21）: 5233 - 5238.

[18] CHI Y, YAO Y, WANG S, et al. Anlotinib for metastasis soft tissue sarcoma: a randomized, double-blind, placebocontrolled and multi-centered clinical trial [J] . J Clin Oncol, 2018, $36: 11503$.

[19] Randrup HC,Grimm D,Bauer J,et al.Effects and side effects of using sorafenib and sunitinib in the treatment of metastatic renal cell carcinoma[J].Inter J Mol Sci,2017,18(2):4611-4625.

[20] Jagodzińska-Mucha P, witaj T,Kozak K,et al.Long-term results of therapy with sunitinib in metastatic alveolar soft part sarcoma[J].Tumori,2017,103(3):231-235.

[21] Li T,Wang L,Wang $\mathrm{H}$,et al. A retrospective analysis of 14 consecutive Chinese patients with unresectable or metastatic alveolar soft part sarcoma treated with sunitinib [J].Invest New Drugs,2016,34(6):1-6.

[22] JAGODZIŃSKA-MUCHA P,ŚWITAJ T, KOZAK K, et al.Long-term results of therapy with sunitinib in metastatic alveolar soft part sarcoma[J]. Tumori, 2017, 103(3):231-235.

[23] CRANMER L D, LOGGERS E T, POLLACK S M. Pazopanib in the management of advanced soft tissue sarcomas[J]. Ther Clin Risk Manag, 2016, 12:941-955.

[24] MINOCHA M, KHURANA V, MITRA A K. Determination of pazopanib(GW-786034)in mouse plasma and brain tissue by liquid chromatography-tandem mass spectrometry(LC/MS-MS)[J]. J Chromatogr B Analyt Technol Biomed Life Sci, 2012,901:85-92.

[25] KIM M, KIM T M, KEAM B, et al. A phase Itrial of pazopanib in patients with metastatic alveolar soft part sarcoma[J].Oncologist, 2019, 24(1):20-e29.

[26] STACCHIOTTI S, MIR O, LE CESNE A, et al. Activity of pazopanib and trabectedin in advanced alveolar soft part sarcoma[J]. Oncologist, 2018, 23(1):62-70.

[27] Judson I, Morden JP, Kilburn L, et al. Cediranib in patients with alveolar soft part sarcoma (CASPS): a double blind, placebo controlled, randomised, phase 2 trial[J]. Lancet Oncol, 2019, 20(7):1023 1034. DOI: 10.1016/s1470 2045(19) 302153.

[28] GLOD J, COHEN J W, WIDEMANN B C, et al. Cediranib phase $\Pi$ study in children with metastatic alveolar soft part sarcoma(ASPS)[J]. J Clin Oncol, 2018, 36(15_suppl):10540.

[29] Zhou Y, Tang F,Wang Y,et al.Advanced alveolar soft part sarcoma responds to apatinib[J].Oncotarget,2017,8(30):5031450322 .

[30] SCHÖFFSKI P, WOZNIAK A, KASPER B, et al. Activity and safety of crizotinib in patients with alveolar soft part sarcoma with rearrangement of TFE3:European Organization for Research and Treatment of Cancer(EORTC)phase $\amalg$ trial90101 
'CREATE'[J]. Ann Oncol, 2018, 29(3):758-765.

[31] Conley AP,Trinh VA,Zobniw CM,et al.Positive tumor response to combined checkpoint inhibitors in a patient with refractory alveolar soft part sarcoma[J].J Global Oncol,2017,4:1-6.

[32] Mo HN, Huang J, Xu JC, et al. Safety, anti-tumour activity, and pharmacokinetics of fixed-dose SHR-1210, an anti-PD-1 antibody in advanced solid tumours: a dose-escalation, phase 1 study[ J]. Br J Cancer, 2018, 119(5): 538-545

[33] Guzik K , Tomala M, Muszak D, et al. Development of the inhibitors that target the PD-1/PD-L1 interaction-A brief look at progress on small molecules, peptides and macrocycles[ J]. Molecules, 2019, 24(11): E2071.

[34] Nie J, Wang C, Liu Y, et al. Addition of low-dose decitabine to anti-PD-1 antibody camrelizumab in relapsed/refractory classical Hodgkin lymphoma[J]. J Clin Oncol, 2019, 37 (17): 1479-1489.

[35] Song Y, Wu J, Chen X, et al. A single- arm, multicenter, phase II study of camrelizumab in relapsed or refractory classical hodgkin lymphoma[J]. Clin Cancer Res, 2019, 25 (24): 7363-7369.

[36] Markham A, Keam SJ. Camrelizumab: first global approval[ J]. Drugs, 2019, 79(12): 1355-1361.

[37] Lu X, Wei G, Jie X, et al. Apatinib plus camrelizumab (SHR1210) for unresectable high-grade osteosarcoma (APFAO) progressing after chemotherapy: A prospective, open label, phase $I$ trial[ J]. J Clin Oncol, 2019, 37(15 Suppl): 11013.

[38] Pang Q, Li X, Zhang W. Safety and effect of radiation therapy combined with anti- PD- 1 antibody SHR- 1210 as firstline treatment on patients with intolerable concurrent chemoradiotherapy esophageal cancer: a phase $1 \mathrm{~b}$ clinical trial[J]. Int J Radiat Oncol Biol Phys, 2018, 102(3): E39.

[39] Jing H, Binghe X, Hongnan M, et al. Safety, activity, and biomarkers of SHR-1210, an anti- PD-1 antibody, for patients with advanced esophageal carcinoma[J]. Clin Cancer Res, 2018, 24(6): 1206-1304

[40] Shen L, Peng Z, Zhang YQ. Camrelizumab combined with capecitabine and oxaliplatin followed by camrelizumab and apatinib as first-line therapy for advanced or metastatic gastric or gastroesophageal junction cancer: updated results from a multicenter, open label phase II trial[J]. Journal of clinical oncology : official journal of the American Society of Clinical Oncology, 2019, 37(15): 4031-4031.

[41] Qin SK, Ren ZG, Meng ZQ, et al. A randomized multicentered phase II study to evaluate SHR-1210 (PD-1 antibody) in subjects with advanced hepatocellular carcinoma (HCC) who failed or intolerable to prior systemic treatment[J]. Ann Oncol, 2018, 29: 719-720

[42] XU J, Zhang Y, Jia R, et al. Anti-PD-1 antibody SHR-1210 combined with apatinib for advanced hepatocellular carcinoma, gastric, or esophagogastric junction cancer: an open- label, dose escalation and expansion Study[J]. Clin Cancer Res, 2019, 25(2): 515-523

[43] Zhou C, Gao G, Wu F. A phase Ib study of SHR-1210 plus Apatinib for heavily previously treated advanced nonsquamous non- small cell lung cancer (NSCLC) patients [abstract no. e21017][J]. Journal of clinical oncology: official journal of the American Society of Clinical Oncology, 2018, 36(15): e21017

[44] Kwong DLW. Camrelizumab for nasopharyngeal carcinoma: a new hope?[J]. Lancet Oncol, 2018, 19(10): 1266- 1267.

[45] Postow MA, Sidlow R, Hellmann MD. Immune-related adverse events associated with immune checkpoint blockade [J]. N Engl J Med, 2018, 378(2): 158-168.

[46] Wilky BA, Trucco MM, Subhawong TK, et al. Axitinib plus pembrolizumab in patients with advanced sarcomas including alveolar soft part sarcoma: a single centre, single arm, phase 2 trial[J]. Lancet Oncol, 2019, 20(6): 837848.

[47] LEWIN J, DAVIDSON S, ANDERSON N D, et al. Response to immune checkpoint inhibition in two patients with alveolar softpart sarcoma[J]. Cancer Immunol Res, 2018, 6(9):1001-1007.

[48] YANG S, YANG J, HAN X, et al. Effect of JS001, a monoclonal antibody targeting programed death-1(PD-1), on responses and disease control in patients with advanced or refractory alveolar soft part sarcoma:results from a phase 1 trial[J]. J Clin Oncol, 2018, 36(15_suppl):11572. 


\section{Figures}
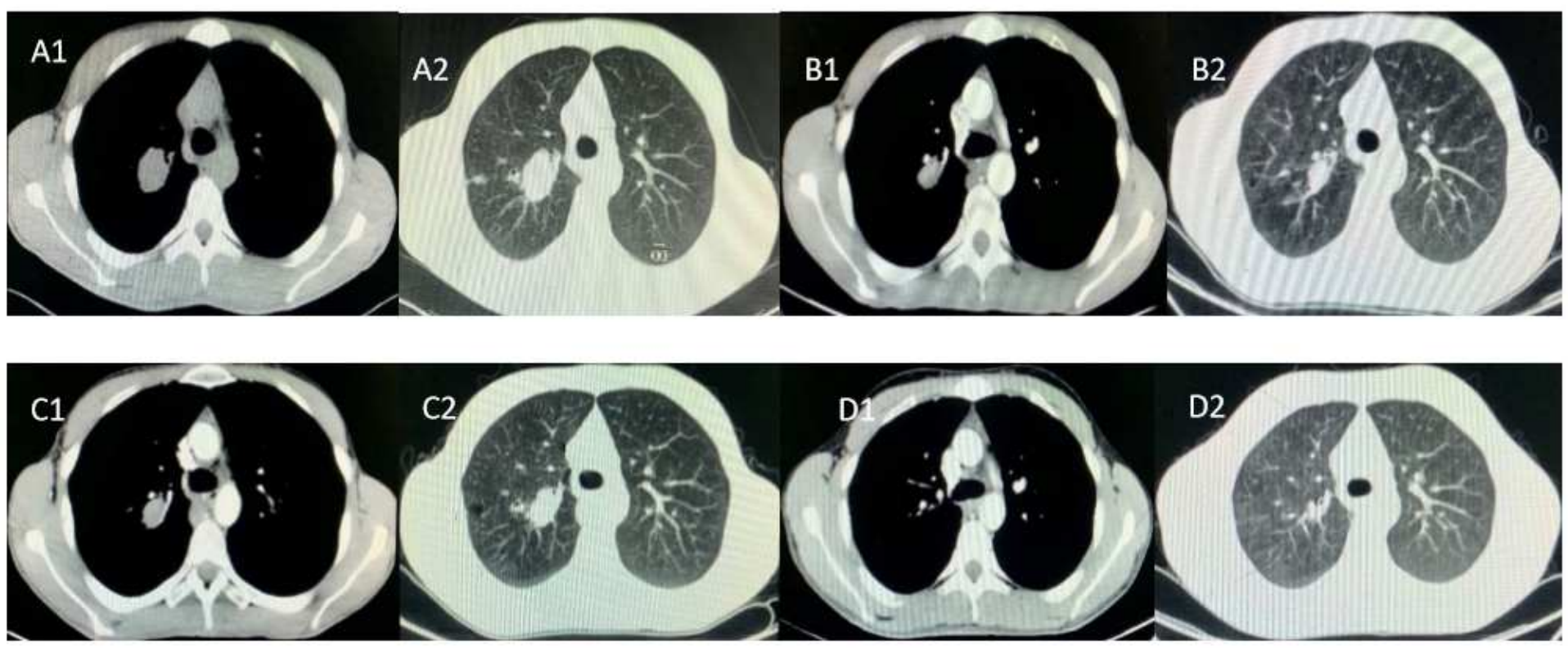

\section{Figure 1}

Dynamic changes in chest CT imaging during treatment A1区2 Before treatment $₫ 2018-08-31 \rrbracket \mathrm{B} 1 \rrbracket 2$ After chemotherapy + apatinib treatment $₫ 2019-05-28 \rrbracket C 1 \otimes 2$ Before anlotinib + camrelizumab treatment $\rrbracket$ 2019-10-24 \D1 \2 After anlotinib + camrelizumab treatment $₫ 2020-9-27 \rrbracket$ 

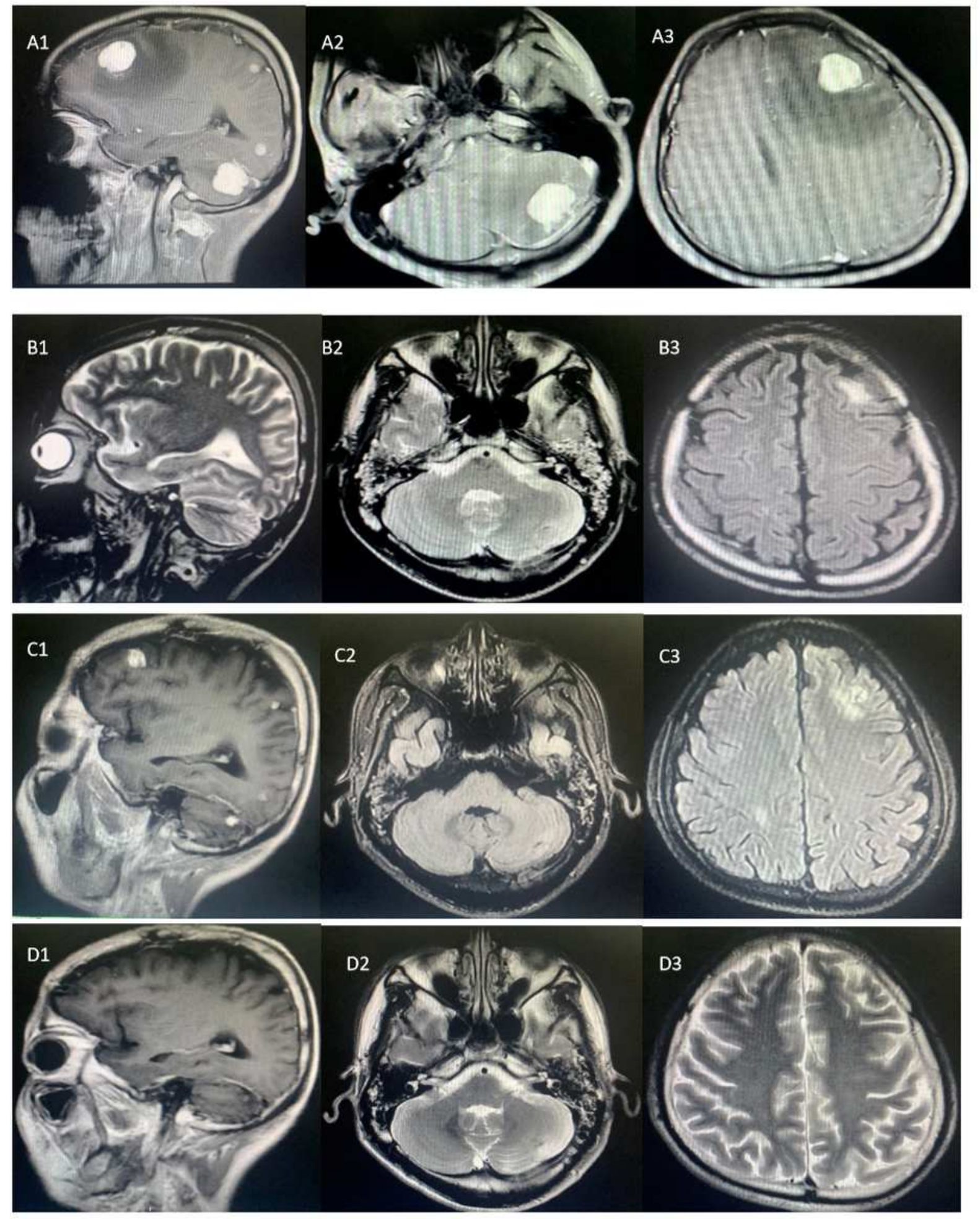

Figure 2

Dynamic changes in brain MRI imaging during treatment $A 1 \rrbracket 2 \rrbracket 3$ Before treatment $₫ 2018-08-31 \rrbracket B 1 \rrbracket 2 \varangle 3$ After chemotherapy + apatinib treatment $₫ 2019-0528 \rrbracket C 1 \otimes 2 \rrbracket 3$ Before anlotinib + camrelizumab treatment $\llbracket 2019-10-24 \llbracket \mathrm{D} 1 \rrbracket 2 \rrbracket 3$ After anlotinib + camrelizumab treatment $₫ 2020-9-27 \rrbracket$ 


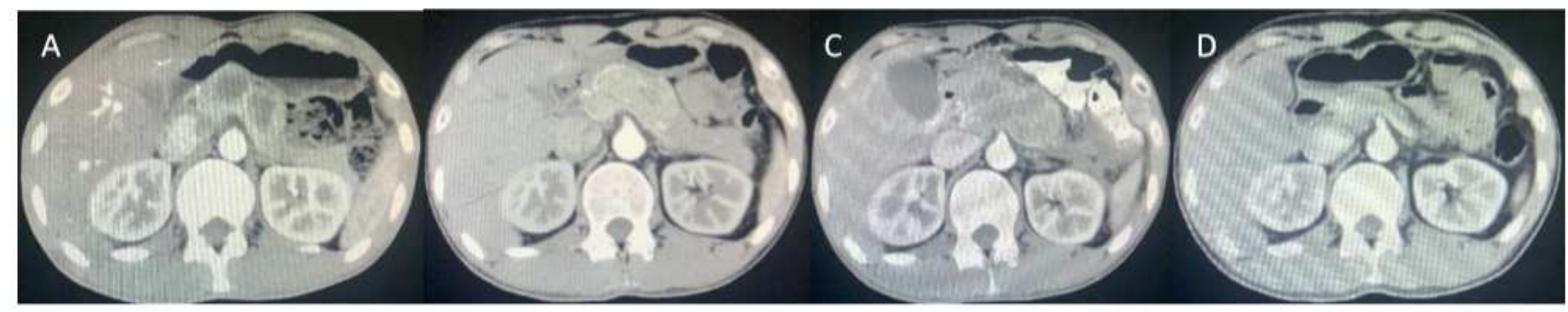

\section{Figure 3}

Dynamic changes in CT imaging of the pancreas during treatment A Before treatment $₫ 2018-10-4 \rrbracket B$ After chemotherapy + apatinib treatment $\varangle 2019-05-28 \rrbracket$ C Before anlotinib + camrelizumab treatment $₫ 2019-10$ 24囚D After anlotinib + camrelizumab treatment $₫ 2020-9-27 \rrbracket$

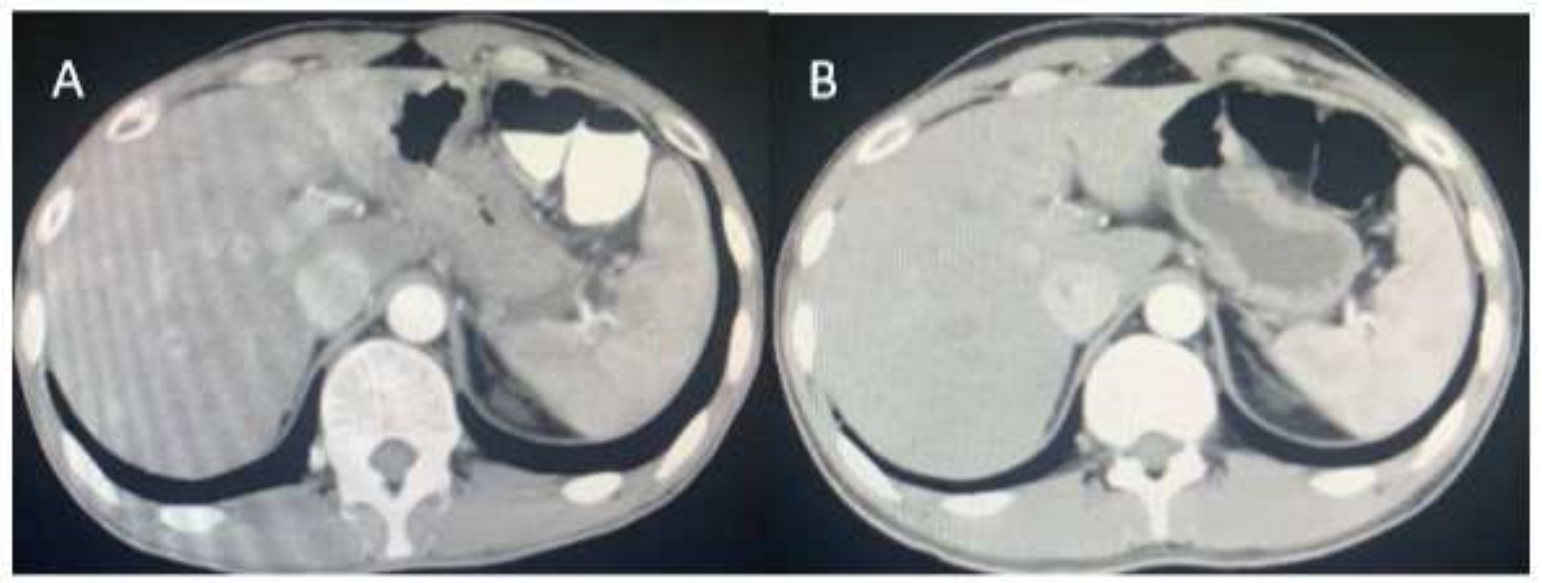

Figure 4

Dynamic changes in CT imaging of the liver during treatment A Before anlotinib + camrelizumab treatment $₫ 2019-10-24 \rrbracket$ B After anlotinib + camrelizumab treatment $₫ 2020-927 \rrbracket$ 